\title{
A Thermal Immiscible Multiphase Flow Simulation by Lattice Boltzmann Method
}

\author{
Wei Gong ${ }^{1}$, Sheng Chen $^{1}$, Yuying Yan ${ }^{* 1,2}$ \\ ${ }^{1}$ Fluids \& Thermal Engineering Group, Faculty of Engineering, University of Nottingham, \\ University Park, Nottingham, United Kingdom \\ ${ }^{2}$ Center for Fluids \& Thermal Engineering, University of Nottingham Ningbo, China \\ *corresponding author: yuying.yan@nottingham.ac.uk
}

\begin{abstract}
The lattice Boltzmann (LB) method, as a mesoscopic approach based on the kinetic theory, has been significantly developed and applied in a variety of fields in the recent decades. Among all the LB community members, the pseudopotential LB plays an increasingly important role in multiphase flow and phase change problems simulation. The thermal immiscible multiphase flow simulation using pseudopotential LB method is studied in this work. The results show that it is difficult to achieve multi-bubble/droplet coexistence due to the unphysical mass transfer phenomenon of "the big eat the small" - the small bubbles/droplets disappear and the big ones getting bigger before a physical coalescence when using an internal energy based temperature equation for single-component multiphase (SCMP) pseudopotential models. In addition, this unphysical effect can be effectively impeded by coupling an entropy-based temperature field, and the influence on density fields with different energy equations are discussed. The findings are identified and reported in this paper for the first time. This work gives a significant inspiration for solving the unphysical mass transfer problem, which determines whether the
\end{abstract} SCMP LB model can be used for multi-bubble/droplet systems.

Keywords: multi-bubble/droplet coexistence, unphysical mass transfer, thermal immiscible multiphase flow, pseudopotential lattice Boltzmann method

\section{Introduction}

As an unconventional numerical method which is based on the mesoscopic kinetic equation, the lattice Boltzmann (LB) method [1-4] has drawn considerable attention and been applied to a wide range of fields such as phase change [5], wetting phenomena [6,7], porous media flow [8], electroosmotic driven flow [9] and flow with moving boundaries [10], etc. The 
pseudopotential LB method, which was firstly presented by Shan and Chen [11,12], has become one of the most important and popular LB community members due to its conceptual simplicity and computational efficiency [4]. In this model, the pseudopotential $\Psi$ is introduced to mimic the interaction forces for the neighbouring particles, which renders an automatic phase separation without a specific tracking or capturing method for the interface between different phases. Owing to this particular phase separation characteristic, the pseudopotential LB has been well applied in multiphase flow and phase change.

The original interaction force of single-component multiphase (SCMP) models acting on a particle at the position $\mathbf{x}$ from the nearest-neighbouring particles is given by [11]

$$
\boldsymbol{F}(\boldsymbol{x}, t)=-G \Psi(\boldsymbol{x}) \sum_{\alpha} w_{\alpha} \Psi\left(\boldsymbol{x}+\boldsymbol{e}_{\alpha} \delta_{t}\right) \boldsymbol{e}_{\alpha},
$$

where $G, w_{\alpha}, \mathbf{e}_{\alpha}$ and $\delta_{t}$ are the parameter controlling the interaction strength, the weights, the lattice speed vector and the lattice time step, respectively. Besides the use of SCMP models, multi-component multiphase (MCMP) models are also widely applied, and the interaction force can be written as [12]

$$
\boldsymbol{F}_{\sigma}(\boldsymbol{x}, t)=-\Psi_{\sigma}(\boldsymbol{x}) \sum_{\bar{\sigma}} G_{\sigma \bar{\sigma}} \sum_{\alpha} w_{\alpha} \Psi_{\bar{\sigma}}\left(\boldsymbol{x}+\boldsymbol{e}_{\alpha} \delta_{t}\right) \boldsymbol{e}_{\alpha}
$$

where $\sigma$ and $\bar{\sigma}$ denote the corresponding components. It has been reported that generally multibubble/droplet configurations cannot be formed when performing a conventional first order multiphase pseudopotential model [3], which impedes an extended application of LB in a number of fields such as atomization, spray formation and phase segregation. Our study finds that the MCMP models do not suffer such a problem, and some multi-bubble/droplet flow studies from other researchers using MCMP models can be found in reference [13-18]. However, the multi-component models have not drawn as much attention as the singlecomponent models because of the density ratio and viscosity issues [3].

Another major finding in our study is that the selection of a proper energy equation for thermal LB models can be advantageous for multi-bubble/droplet systems as well. For thermal LB models, without considering the viscous heat dissipation and the compression work, the internal energy equation is given by [4]

$$
\partial_{t}\left(\rho c_{v} T\right)+\nabla \cdot\left(\rho c_{v} T \boldsymbol{v}\right)=\nabla \cdot(\lambda \nabla T)
$$


where $\rho$ is the density, $c_{v}$ is the specific heat capacity, $T$ is the temperature, $\mathbf{v}$ is the velocity vector, $\lambda$ is thermal conductivity and $t$ denotes the time. Furthermore, if $c_{v}$ is a constant, the internal energy equation can be reduced to

$$
\partial_{t} T+v \cdot \nabla T=\frac{1}{\rho c_{v}} \nabla \cdot(\lambda \nabla T)
$$

Li Q. used another energy equation in their model [5]

$$
\partial_{t} T+\boldsymbol{v} \cdot \nabla T=\frac{1}{\rho c_{v}} \nabla \cdot(\lambda \nabla T)-\frac{T}{\rho c_{v}}\left(\frac{\partial p_{E O S}}{\partial T}\right)_{\rho} \nabla \cdot \boldsymbol{v}
$$

where $p_{E O S}$ is the non-ideal equation of state, and this energy equation is derived from the entropy equation neglecting the viscous heat dissipation

$$
\rho T \frac{D s}{D t}=\nabla \cdot(\lambda \nabla T)
$$

with the thermodynamic relation

$$
T d s=c_{v} d T+T\left(\frac{\partial p_{E O S}}{\partial T}\right)_{\rho} d\left(\frac{1}{\rho}\right)
$$

where $s$ represents the entropy. In addition, in their work it has also been reported that the thermal lattice scheme can produce a significant error by the forcing term, thus in this work all the thermal equation are solved by the classical fourth-order Runge-Kutta scheme [19].

Through studying thermal LB models using the two energy equations (4) and (5), it is found that it is much more difficult to form a multi-bubble/droplet configuration when applying Eq. (4). The fact of unachievable multi-bubble/droplet configuration is due to an unphysical mass transfer phenomenon which we call "the big eat the small". As shown in Fig. 1, the small bubbles/droplets become smaller and even disappear over time while the big ones getting bigger without a physical coalescence, simulated using the basic Shan-Chen SCMP model [12]. It should be pointed out that an isothermal system is equal to a thermal LB model along with the energy equation (4), which means the isothermal SCMP models also suffer the same unphysical mass transfer problem.

\section{Take in Fig. 1}




\section{Numerical simulation}

The original Shan-Chen model [12], an improved Bhatnagar-Gross-Krook (BGK) model with the given code in reference [20], the Gong-Cheng model [21], an improved multiple-relaxationtime (MRT) model from Li Q. [22] and the midrange repulsion model [23], all of which involve various forcing schemes, non-ideal equation of states and collision forms, are all tested in our study. The result shows none of the model can get rid of the unphysical mass transfer when coupling the energy equation (4). The following numerical simulation and discussion are based on the Li Q. improved MRT model and the details of the model can be found in reference [5]. Following their model, the boundary conditions are set as Zou-He solid boundaries for the top and the bottom and the periodical boundaries for the left and the right to close a $200 \times 100$ computational region. For the thermal simulation, the top and bottom boundaries are constant temperature $T_{s}$ and $T_{b}$ with a small temperature difference $\Delta T=T_{b}-T_{s}=0.001$ for a simple heat transfer case. It should be noticed that it is the energy equation selection that matters in our study other than the heat transfer cases. First, a single bubble with diameter 40 is simulated to test the coexistence of liquid/vapour densities by the Maxwell construction with the saturation temperature $T_{s}=0.86 T_{c}$, where $T_{c}$ is the critical temperature. The coexistence densities and the Maxwell construction using Ping-Robinson EOS [24] are shown in Fig. 2. From the picture it can be seen the simulation result is in good agreement with the theory thermodynamic consistency.

\section{Take in Fig. 2}

Bubble systems and droplet systems are tested with an initial larger bubble/droplet with diameter 40 and a small one with diameter 20 using the two different energy equations. The radius variation curves of the small bubble/droplets are shown in Fig. 3. From the figure it can be seen that the unphysical mass transfer is much severer in bubble systems than droplet systems, and the mass transfers faster when the bubble/droplet volume ratio increases. Besides, the mass transfer only occurs from the smaller bubble/droplets to the larger ones, but not vice versa. In addition, the problem can be effectively restrained by coupling the entropy-based energy equation.

Take in Fig. 3 
Finally in Fig. 4 the density fields for the two different energy equations are compared. The main difference of the two density fields is that when conducting Eq. (5), one layer of high density region appears outside of the bubble/droplet interface, where for Eq. (4) it should be the same with liquid/vapour density. This spurious density layer caused the interface nonmonotonic. If the unphysical mass transfer is caused by the spurious currents, this highdensity layer might be the reason that disturbs the spurious currents and thereby restrains the phenomenon of "the big eat the small". Nevertheless, the real reason and the way to eliminate the unphysical phenomenon for SCMP models still need to be investigated in the future work.

\section{Take in Fig. 4}

\section{Conclusions}

In the present study, we report our new findings of a critical problem of the conventional thermal/isothermal SCMP pseudopotential LB models. It is the first time this type of problem is identified and reported. After testing a number of typical models involving various forcing schemes, non-ideal equation of states and the relaxation schemes of SCMP LB models with the internal energy based energy equation, we found the multiple bubbles/droplets could not coexist due to the unphysical mass transfer phenomenon, or "the big eat the small". Meanwhile, this unphysical problem can be effectively restrained with an entropy-based energy equation. Although the mass transfer problem cannot be solved in this work, our study points out a reasonable orientation for the further investigation. Form our study the following conclusions can be drawn:

(1) The unphysical mass transfer phenomenon exists in most of the thermal SCMP pseudopotential LB models with the presented internal energy based energy equation, of which the isothermal situation is included.

(2) The mass transfer only occurs from the smaller bubble/droplets to the larger ones, but not vice versa.

(3) The unphysical mass transfer is much severer in bubble systems than droplet systems, and the mass transfers faster when the bubble/droplet volume ratio increases. 
(4) The unphysical mass transfer can be effectively impeded by coupling an entropy-based energy equation.

(5) A high-density layer outside of the bubble/droplet is produced by coupling the entropybased energy equation, which might be the reason to restrain the unphysical phenomenon.

\section{Acknowledgement}

The authors would like to acknowledge the financial support of this work by the doctoral degree scholarship of China Scholarship Council (CSC) for Mr Wei Gong and the University of Nottingham, UK, for Yuying Yan and Wei Gong, and this work is also supported by Ningbo Science and Technology Bureau Technology Innovation Team project under Grant No. 2016B10010.

\section{References}

[1] Chen, S.; Doolen, G. D. Lattice Boltzmann method for fluid flows. Annual review of fluid mechanics, 1998, 30, 329-364.

[2] Swift, M. R.; Osborn, W.; Yeomans, J. Lattice Boltzmann simulation of nonideal fluids. Physical Review Letters, 1995, 75, 830.

[3] Chen, L.; Kang, Q.; Mu, Y.; He, Y.-L.; Tao, W.-Q. A critical review of the pseudopotential multiphase lattice Boltzmann model: Methods and applications. International Journal of Heat and Mass Transfer, 2014, 76, 210-236.

[4] Li, Q.; Luo, K. H.; Kang, Q. J.; He, Y. L.; Chen, Q.; Liu, Q. Lattice Boltzmann methods for multiphase flow and phase-change heat transfer. Progress in Energy and Combustion Science, 2015, 10.1016/j.pecs.2015.10.001.

[5] Li, Q.; Kang, Q. J.; Francois, M. M.; He, Y. L.; Luo, K. H. Lattice Boltzmann modeling of boiling heat transfer: The boiling curve and the effects of wettability. International Journal of Heat and Mass Transfer, 2015, 85, 787-796.

[6] Gong, W.; Zu, Y.; Chen, S.; Yan, Y. Wetting transition energy curves for a droplet on a square-post patterned surface. Science Bulletin, 2017, 62, 136-142.

[7] Gong, W.; Yan, Y.; Chen, S.; Giddings, D. Numerical Study of Wetting Transitions on Biomimetic Surfaces Using a Lattice Boltzmann Approach with Large Density Ratio. Journal of Bionic Engineering, 2017, 14, 486-496. 
[8] Chen, S.; Yang, B.; Zheng, C. A lattice Boltzmann model for heat transfer in porous media. International Journal of Heat and Mass Transfer, 2017, 111, 1019-1022.

[9] Li, B.; Zhou, W. N.; Yan, Y. Y.; Tian, C. Evaluation of electro-osmotic pumping effect on microporous media flow. Applied Thermal Engineering, 2013, 60, 449-455.

[10] Yan, Y. Y.; Zu, Y. Q. Numerical simulation of heat transfer and fluid flow past a rotating isothermal cylinder - A LBM approach. International Journal of Heat and Mass Transfer, 2008, 51, 2519-2536.

[11] Shan, X.; Chen, H. Simulation of nonideal gases and liquid-gas phase transitions by the lattice Boltzmann equation. Physical Review E, 1994, 49, 2941-2948.

[12] Shan, X.; Chen, H. Lattice Boltzmann model for simulating flows with multiple phases and components. Physical Review E, 1993, 47, 1815-1819.

[13] Sehgal, B.; Nourgaliev, R.; Dinh, T. Numerical simulation of droplet deformation and break-up by lattice-Boltzmann method. Progress in Nuclear Energy, 1999, 34, 471-488.

[14] Nekovee, M.; Coveney, P. V.; Chen, H.; Boghosian, B. M. Lattice-Boltzmann model for interacting amphiphilic fluids. Physical Review E, 2000, 62, 8282.

[15] Dörfler, F.; Rauscher, M.; Koplik, J.; Harting, J.; Dietrich, S. Micro- and nanoscale fluid flow on chemical channels. Soft Matter, 2012, 8, 9221.

[16] Gupta, A.; Kumar, R. Lattice Boltzmann simulation to study multiple bubble dynamics. International Journal of Heat and Mass Transfer, 2008, 51, 5192-5203.

[17] Yu, Z.; Yang, H.; Fan, L.-S. Numerical simulation of bubble interactions using an adaptive lattice Boltzmann method. Chemical Engineering Science, 2011, 66, 3441-3451.

[18] Zhang, Q.; Sun, D.; Zhang, Y.; Zhu, M. Lattice Boltzmann modeling of droplet condensation on superhydrophobic nanoarrays. Langmuir, 2014, 30, 12559-12569.

[19] Liu, H.; Valocchi, A. J.; Zhang, Y.; Kang, Q. Phase-field-based lattice Boltzmann finite-difference model for simulating thermocapillary flows. Physical Review E, $2013,87,013010$.

[20] Huang, H.; Sukop, M.; Lu, X.: Multiphase lattice Boltzmann methods: Theory and application; John Wiley \& Sons, 2015. 
[21] Gong, S.; Cheng, P. Numerical investigation of droplet motion and coalescence by an improved lattice Boltzmann model for phase transitions and multiphase flows. Computers \& Fluids, 2012, 53, 93-104.

[22] Li, Q.; Luo, K. H.; Li, X. J. Lattice Boltzmann modeling of multiphase flows at large density ratio with an improved pseudopotential model. Physical Review. E: Statistical, Nonlinear, and Soft Matter Physics, 2013, 87, 053301.

[23] Chibbaro, S.; Falcucci, G.; Chiatti, G.; Chen, H.; Shan, X.; Succi, S. Lattice Boltzmann models for nonideal fluids with arrested phase-separation. Physical Review. E: Statistical, Nonlinear, and Soft Matter Physics, 2008, 77, 036705.

[24] Yuan, P.; Schaefer, L. Equations of state in a lattice Boltzmann model. Physics of Fluids, 2006, 18, 042101. 


\section{Figures:}

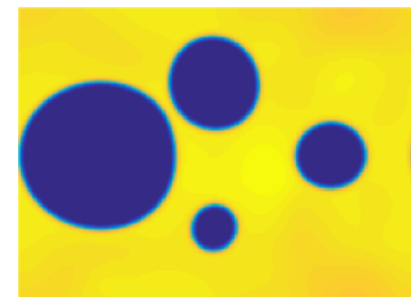

(a)

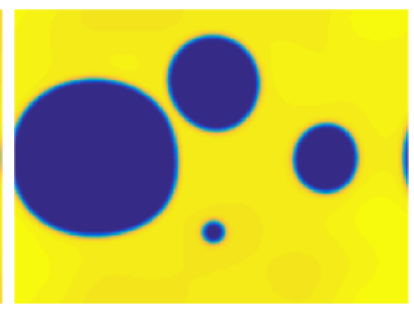

(b)

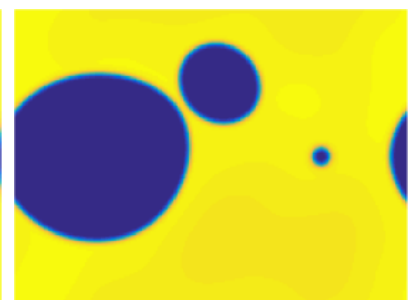

(c)

Fig. 1 Multi-bubble configuration evolution in a $200 \times 150$ computational domain with periodical boundary condition (a) $t=200 \delta_{t}$ (b) $t=400 \delta_{t}$ (c) $t=800 \delta_{t}$

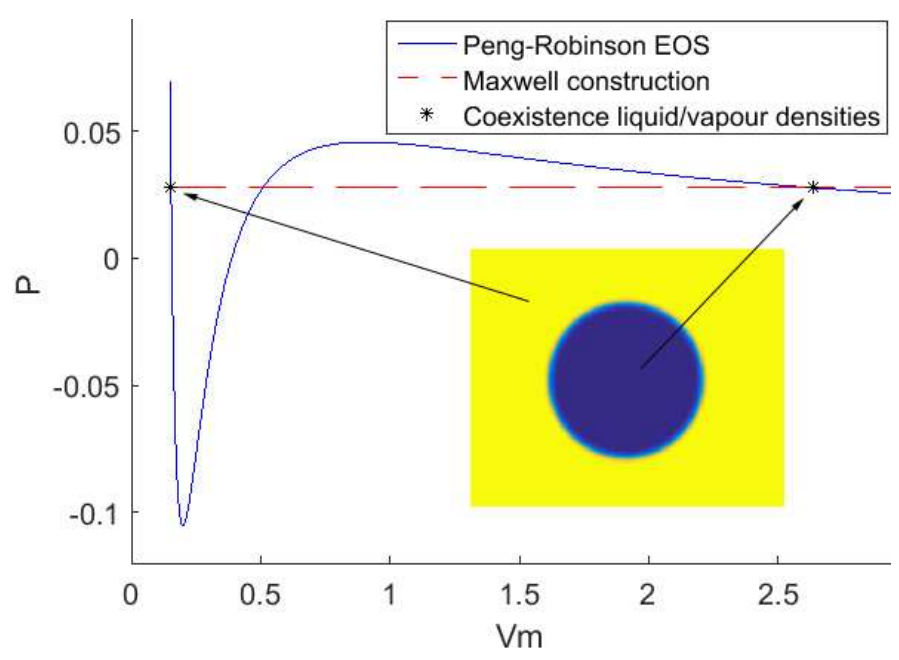

Fig. 2 The Maxwell construction with $T_{s}=0.86 T_{c}$ for Ping-Robinson EOS

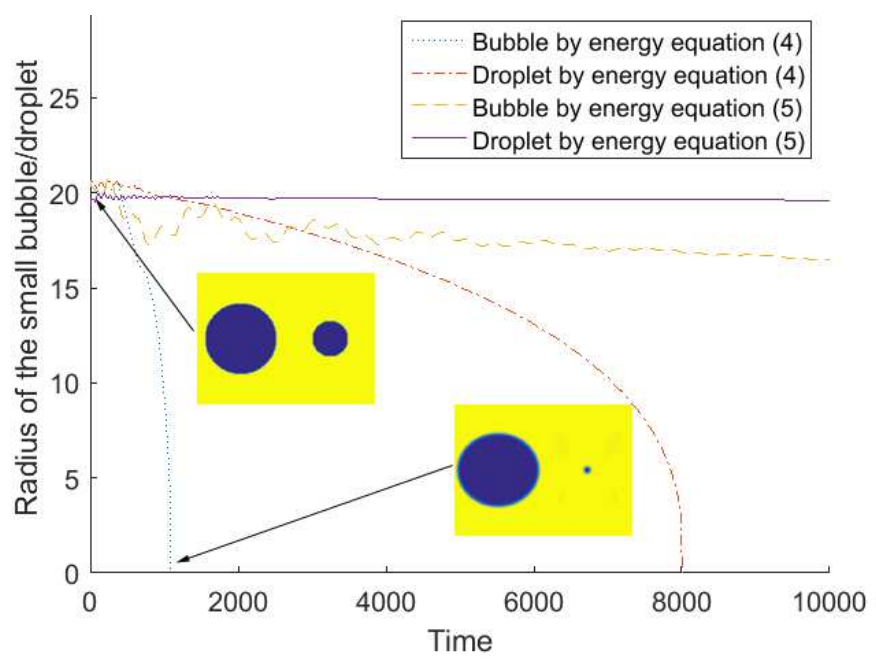

Fig. 3 The small bubble/droplets variations with different energy equations 


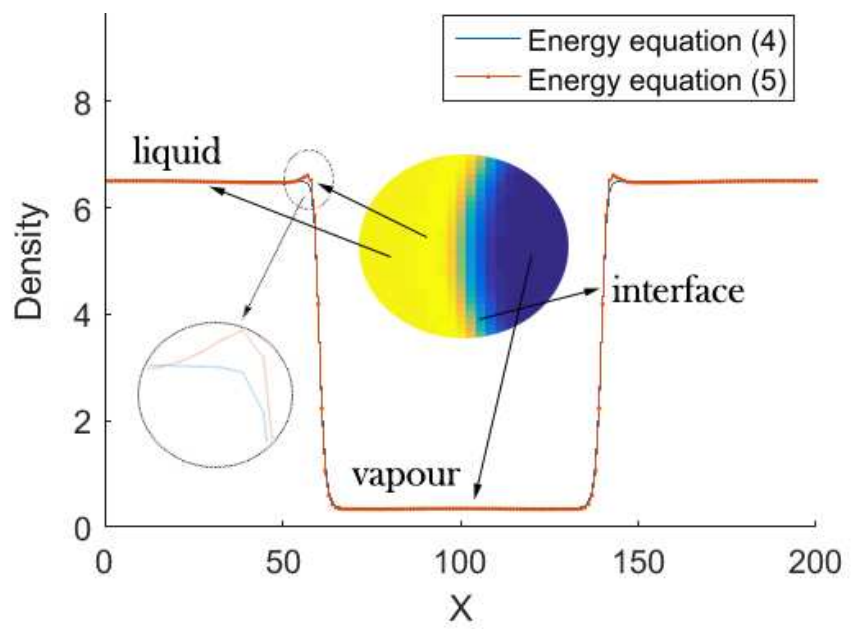

Fig. 4 The density variation in the cross section with different energy equations 